\title{
Pharmacogenetics of SLCO1B1: haplotypes, htSNPs and hepatic expression in three distinct Asian populations
}

\author{
Srinivasa Rao Jada • Shu Xiaochen • Liu Yan Yan • \\ Xiang Xiaoqiang • Suman Lal $\cdot$ Shu Feng Zhou • \\ London Lucien Ooi • Balram Chowbay
}

Published online: 21 June 2007

(C) Springer-Verlag 2007

An error occurred in Table 1. The data in line 12 was incorrect. See the correct version of Table 1 below.

The online version of the original article can be found at http://dx.doi. org/10.1007/s00228-007-0285-5.

S. R. Jada $\cdot$ S. Xiaochen $\cdot$ L. Y. Yan $\cdot$ X. Xiaoqiang $\cdot$ S. Lal $\cdot$

B. Chowbay $(\bowtie)$

Laboratory of Clinical Pharmacology,

Division of Medical Sciences, National Cancer Centre,

11 Hospital Drive,

Singapore 169610, Singapore

e-mail: ctebal@nccs.com.sg

\section{S. F. Zhou}

School of Life Sciences, Faculty of Science,

Queensland University of Technology,

2 George Street,

Brisbane, Queensland 4001, Australia

L. L. Ooi

Department of Surgical Oncology, National Cancer Centre,

11 Hospital Drive,

Singapore 169610, Singapore 
Table 1 Primers and PCR conditions used for sequence analysis

\begin{tabular}{|c|c|c|c|c|c|}
\hline SLCO1B1 SNP & Location & Primer Sequence ${ }^{a}$ & $\begin{array}{l}\text { Fragment } \\
\text { size (bp) }\end{array}$ & $\begin{array}{l}\text { Annealing } \\
\text { temperature } \\
\left({ }^{\circ} \mathrm{C}\right)\end{array}$ & $\begin{array}{l}\text { Amplification } \\
\text { cycles }\end{array}$ \\
\hline g. $-11187 \mathrm{G}>A$, g. $-11110 \mathrm{~T}>\mathrm{G}$ & Promoter & $\begin{array}{l}\text { F-5'-GGATTGCTATTTTGACAACT-3' } \\
\text { R-5'-CTGCAAGACCATTTTTCTGA-3' }\end{array}$ & 681 & 51.0 & 30 \\
\hline g. $-10499 \mathrm{~A}>\mathrm{C}$, g. $-314 \mathrm{~T}>\mathrm{C}$ & Promoter & $\begin{array}{l}\text { F-5'-GTCAATAAAAGGAAACTGTG-3' } \\
\text { R-5'-CTTCCGTGAAAATTTCTTAC-3' }\end{array}$ & 668 & 52.8 & 35 \\
\hline c. $217 \mathrm{~T}>\mathrm{C}$ & Exon 2 & $\begin{array}{l}\text { F-5'-GCAGCTCTGTCACTCAGCTT-3' } \\
\text { R-5'-ACCTTTAGATACCAGTAGCAC-3' }\end{array}$ & 321 & 55.3 & 35 \\
\hline c. $245 \mathrm{~T}>\mathrm{C}$ & Exon 3 & $\begin{array}{l}\text { F-5'-CAATGAGTGGTCTAATGTAG-3' } \\
\text { R-5'-ACAAGGTACTGATAGTGGCA-3' }\end{array}$ & 348 & 50.0 & 30 \\
\hline $\begin{array}{l}\text { c. } 388 \mathrm{~A}>\mathrm{G}, \text { c. } 411 \mathrm{G}>\mathrm{A}, \text { c } .452 \mathrm{~A}>\mathrm{G} \\
\text { c. } 455 \mathrm{G}>\mathrm{A}, \text { c } .463 \mathrm{C}>\mathrm{A}, \text { c. } 467 \mathrm{~A}>\mathrm{G}\end{array}$ & Exon 4 & $\begin{array}{l}\text { F-5'-CTGGTAATTTGGGGAAGATAA-3' } \\
\text { R-5'-TAATGGGCGAACTGTGTATAT-3' }\end{array}$ & 407 & 56.7 & 35 \\
\hline c. $521 \mathrm{~T}>\mathrm{C}$, c. $571 \mathrm{~T}>\mathrm{C}$, c. $.597 \mathrm{C}>\mathrm{T}$ & Exon 5 & $\begin{array}{l}\text { F-5'-CACCATATTGTCAAAGTTTGC-3' } \\
\text { R-5'-GCAATTTTACTAGATGCAAGAA-3' }\end{array}$ & 478 & 56.7 & 35 \\
\hline c. $721 \mathrm{G}>\mathrm{A}$ & Exon 6 & $\begin{array}{l}\text { F-5'-ATTAGAACA TATATTTGGGT-3' } \\
\text { R-5'-TTTCCTACTTGTAACTTTC-3' }\end{array}$ & 344 & 45.0 & 30 \\
\hline c. $1007 \mathrm{C}>\mathrm{G}, \mathrm{c} .1058 \mathrm{~T}>\mathrm{C}$ & Exon 8 & $\begin{array}{l}\text { F-5'-TGGTATTGCAGGCTATTCTC-3' } \\
\text { R-5'-TAAAATACCACTTGGAATACAG-3' }\end{array}$ & 309 & 50.3 & 30 \\
\hline c. $1294 \mathrm{~A}>\mathrm{G}$ & Exon 9 & $\begin{array}{l}\text { F-5'-GCAGCTCTGTCACTCAGCTT-3' } \\
\text { R-5'-TATGTTGCTTCTCTTTAGTGG-3' }\end{array}$ & 378 & 54.0 & 31 \\
\hline $\begin{array}{l}\text { c.TTT-DEL, c. } 1385 \mathrm{~A}>\mathrm{G} \text {, } \\
\text { c. } 1454 \mathrm{G}>\mathrm{T}, \text { c. } 1463 \mathrm{G}>\mathrm{C}\end{array}$ & Exon 10 & $\begin{array}{l}\text { F-5'-CTACTTTTTTTCCCTCTTTCTC-3' } \\
\text { R-5'-AATGGAGTCCTTAGTTACAGA-3' }\end{array}$ & 374 & 55.3 & 35 \\
\hline $\begin{array}{l}\text { c. } 1964 \mathrm{~A}>\mathrm{G}, \text { c. } 2000 \mathrm{~A}>\mathrm{G} \\
\text { c. } 2040 \mathrm{~A}>\mathrm{G}\end{array}$ & Exon 14 & $\begin{array}{l}\text { F-5'-TTGGGCTTGTCTTCAATGTT-3' } \\
\text { R-5'-CTTAGTGAAAGGACCAGGAA-3' }\end{array}$ & 300 & 52.3 & 37 \\
\hline
\end{tabular}

${ }^{\mathrm{a}} \mathrm{F}$, Forward primer; $\mathrm{R}$, reverse primer 